\title{
MODELAGEM E PROGNOSE EM POVOAMENTOS NÃO DESBASTADOS DE CLONES DE EUCALIPTO ${ }^{1}$
}

\author{
Marcio Leles Romarco de Oliveira ${ }^{2}$, Helio Garcia Leite ${ }^{3}$, Gilciano Saraiva Nogueira² e João Carlos \\ Chagas Campos ${ }^{3}$
}

\begin{abstract}
RESUMO - Este estudo objetivou desenvolver e propor um procedimento para modelagem do crescimento e produção de plantios clonais de eucalipto não desbastados. Os dados utilizados foram provenientes de 37 clones distribuídos em 4.325 parcelas permanentes de inventários florestais contínuos, sendo cada clone contendo pelo menos três medições com periodicidade anual. Foram estudadas cinco alternativas para avaliação da prognose de clones com mais de três medições de parcela permanentes. Para fins de prognose de estoques volumétricos, o modelo ajustado por estrato de modelagem foi mais eficiente do que o modelo de Clutter. Assim, foi possível concluir que estimativas de crescimento e produção de clones que possuam menos de três medições podem ser realizadas através de modelos específicos por estrato ou de um modelo geral, porém essa decisão deve ser avaliada pelo modelador e pelo usuário, sendo necessária grande interação entre eles.
\end{abstract}

Palavras-chave: Parcelas permanentes, modelo de crescimento e produção.

\section{MODELING AND PROGNOSIS IN NONTHINNED STANDS OF EUCALYPTUS CLONES}

\begin{abstract}
The objective of this work was to develop and propose a procedure for modeling growth and yield of nonthinned eucalyptus clone plantations. The data used were obtained from 37 clones distributed in 4325 permanent plots from continuous forest inventories. Each clone contained at least three annual measurements. Five alternatives were studied for prognosis evaluation of clones with more than three permanent plot measurements. For the volumetric stock prognosis, the study showed that the model adjusted by modeling stratum was more efficient than the Clutter model. It could be concluded that growth and yield estimations for clones with less than three measurements can be performed using specific models per stratum or a general model. Such decision must, however, be evaluated by the modeling agent and the user, and a strong interaction between them is necessary.
\end{abstract}

Keywords: Permanent plots, growth and yield model.

\section{INTRODUÇÃO}

A modelagem de crescimento e produção de povoamentos de eucalipto teve início efetivo no Brasil na década de 1980. Uma das principais contribuições foi a primeira dissertação de mestrado na área de
Mensuração Florestal defendida no Brasil, em 1985, por Trevizol Junior. Naquela ocasião, foi construído um modelo de crescimento e produção $(M C P)$ para plantações de Eucalyptus grandis. Esse modelo foi construído a partir das relações funcionais e fundamentação teórica encontradas em Clutter (1963),

\footnotetext{
${ }^{1}$ Recebido em 03-10-2007 e aceito para publicação em 23.06.2009.

${ }^{2}$ Departamento e Engenharia Florestal da Universidade Federal dos Vales do Jequitinhonha e Mucuri, UFVJM. E-mail: <marcioromarco@gmail.com>e <nogueirags@yahoo.com.br>.

${ }^{3}$ Departamento de Engenharia Florestal da UFV. E-mail: <hgleite@gmail.com>.
} 
dentre outros, sendo utilizados dados de parcelas permanentes de povoamentos localizados na região de Bom Despacho, Minas Gerais. A partir daí, vários modelos foram construídos para diferentes espécies de Eucalyptus e de Pinus, em diferentes regiões do país.

Em anos seguintes, vários autores, trabalhando principalmente com plantios de sementes de eucalipto, construíram modelos do tipo povoamento total, podendo citar Campos et al. (1986), Campos et al. (1988), Pece De Rios (1993) e Rosas (1994). Modelos de distribuição diamétrica foram ajustados por Leite (1990), Guimarães (1994), Nogueira (2005), entre outros, tendo este último autor trabalhado com dados de clones de eucalipto. A maioria dos modelos ajustados a partir da década de 1980 até 2007 foi de modelos compatíveis, do tipo povoamento total e de densidade variável.

Conforme Avery e Burkhart (1994), as decisões de manejo florestal são baseadas em informações sobre condições de recursos atuais e futuros. Os inventários florestais fornecem informações, em um instante no tempo, sobre volume e estatísticas relacionadas. Sendo as florestas sistemas biológicos dinâmicos que estão continuamente mudando, os $M C P$ são necessários para projetar essas mudanças e obter informações para o manejo florestal. Essa dinâmica é ainda maior no caso de povoamentos de clones de eucalipto. A cada ano novos clones são lançados em escala comercial, enquanto outros são excluídos da lista de clones da empresa. Isso resulta em dificuldades na modelagem, uma vez que dados passados em determinados momentos têm que ser descartados em decorrência de uma nova realidade (novos pacotes tecnológicos).

Apesar do avanço científico ocorrido em modelagem, no Brasil existem poucos modelos desenvolvidos para povoamentos de clones de eucalipto.

Uma metodologia para construir um $M C P$ envolve a definição do problema, a obtenção dos dados, a construção do modelo matemático, a aplicação de testes de validação do modelo e a sua aplicação. No caso de povoamentos de clones, pelos motivos mencionados a obtenção dos dados, a construção do modelo e sua aplicação ainda não são triviais.

Na maioria das empresas florestais é ajustado um modelo com maior número de variáveis para todos, ou grande parte, dos clones, demandando maior número de dados, em comparação com um ajuste de um modelo com menor número de variáveis dependente, para um grupo menor de clones com tendências de crescimento semelhante.

Diante dessas considerações e do significativo aumento de plantios de clones de eucalipto no Brasil, foi proposto este estudo, com o objetivo de desenvolver e propor um procedimento para modelagem do crescimento e da produção.

\section{MATERIAL E MÉTODOS}

\subsection{Descrição dos Dados}

Os dados utilizados neste trabalho foram provenientes de 4.325 parcelas permanentes de inventários florestais contínuos realizados entre os anos de 1996 e 2006, em povoamentos de clones de eucalipto, no Estado da Bahia, compreendendo cerca de 70.000 ha de floresta

Foram utilizados dados de 37 clones, distribuídos em 4.325 parcelas permanentes, cada clone contendo pelo menos três medições com periodicidade anual.

Em todas as parcelas mensuradas, em todos os anos foram determinados: a altura total média das árvores dominantes $(H d)$, a área basal $(B)$, o diâmetro médio $(q)$ e o volume comercial com casca $(V)$. Os dados foram submetidos a uma criteriosa consistência, seguindo-se algumas recomendações encontradas em Curtis e Marshall (2005) e Campos e Leite (2006). Alguns dados de parcelas foram eliminados por se tratar de outliers (DRAPER e SMITH, 1998). Algumas parcelas foram eliminadas para compatibilizar a frequência de parcelas permanentes, seguindo-se as condições encontradas no povoamento, e assim obter tendências consistentes para fins de modelagem.

\subsection{Modelagem do crescimento e da produção}

A estratificação para fins de ajuste de modelos de crescimento e produção foi determinada utilizando estatística multivariada. Em cada clone foi ajustado o modelo de Schumacher (1939), $\operatorname{Ln} Y=\beta_{0}+\beta_{I}^{-1}+E$, para as variáveis dependentes: área basal $(B)$, altura dominante $(H d)$, diâmetro médio $(q)$ e volume comercial com casca $(V)$, em função da idade, em meses. Com os oito parâmetros estimados para todos os clones, o próximo passo foi procurar estratificá-los, realizando uma análise de agrupamento pelo método de Tocher (GARCIA e LEITE, 2006). 
Em cada um dos estratos, o método da curva-guia foi utilizado para determinação dos índices de local (CLUTTER et al., 1983), sendo utilizado modelo logístico, conforme Draper e Smith (1998).

\subsection{Modelagem volumétrica e análises de clones com mais de três medições}

Para estimar crescimento e produção para estratos com mais de três medições de parcelas permanentes, foram avaliadas cinco alternativas. A primeira (alternativa 1) foi o ajuste do modelo de Clutter (1963), conforme apresentado a seguir:

$$
\left\{\begin{array}{l}
\operatorname{Ln} B_{2}=\operatorname{Ln} B_{1}\left(\frac{I_{1}}{I_{2}}\right)+\alpha_{1}\left(1-\frac{I_{1}}{I_{2}}\right)+\alpha_{2}\left(1-\frac{I_{1}}{I_{2}}\right) S+\varepsilon \\
\operatorname{Ln} V_{2}=\beta_{0}+\beta_{1} \frac{1}{I_{2}}+\beta_{2} S+\beta_{3} \operatorname{Ln} B_{2}+\varepsilon
\end{array}\right.
$$

em que $I_{1}=$ idade atual, em meses; $I_{2}=$ idade futura, em meses; $B_{1}=$ área basal na idade $I_{1}$, em m².ha $\mathrm{ma}^{-1}$; $B_{2}$ = área basal na idade $I_{2}$, em $\mathrm{m}^{2} \cdot$ ha $^{-1} ; S=$ índice de local, em m; $V_{2}=$ volume $\mathrm{em} \mathrm{m}^{3} \mathrm{ha}^{-1} ; L n=$ logaritmo neperiano; $\beta_{0}, \beta_{1}, \beta_{2}, \beta_{3}, \alpha_{1}$ e $\alpha_{2}=$ parâmetros do modelo; e $\mathcal{E}=$ erro aleatório, sendo $\mathcal{E} \sim \mathrm{N}\left(0, \mathrm{o}^{2}\right)$.

Essas relações funcionais, originalmente estabelecidas para Pinus taeda por Clutter (1963), foram ajustadas aos dados dos 37 clones, como um único estrato. O ajuste foi feito pelo método de mínimos quadrados, em dois estágios.

A segunda alternativa (alternativa 2) consistiu no ajuste do modelo de Clutter, porém com a transformação da expressão da área basal, com o objetivo de compatibilizar o volume observado e o estimado na primeira medição do inventário florestal contínuo. Para que as estimativas das equações ajustadas passassem pelos valores de volumes observados por ocasião do primeiro inventário, foi definida a seguinte expressão de área basal compatível:

$$
B_{1}=\operatorname{Exp}\left(\hat{\beta}_{3}^{-1} \operatorname{Ln} V_{1}-\hat{\beta_{0}}+\hat{\beta}_{1} I_{1}^{-1}+\hat{\beta}_{2} S_{1}\right)
$$

em que $V_{1}, S_{1}$ e $I_{1}$ são, respectivamente, o volume, o índice de local e a idade observados na idade atual (input para prognose); $B_{1}$ é a área basal compatível $\operatorname{com} V_{1}, S_{1}$ e $I_{1}$; e $\hat{\beta_{i}}$ são as estimativas obtidas ao se ajustar o modelo 2. Essa expressão permite encontrar a área basal compatível com a estimativa de volume observada no último inventário com a estimativa do $M C P$.

Dessa forma, utilizando o valor de $L n V$ observado, obtido do primeiro inventário, foi possível encontrar a área basal compatível com a estimativa desse volume, contemplando o modelo de crescimento e produção.

$\mathrm{Na}$ terceira alternativa (alternativa 3) foram testados os modelos sigmoidais logísticos, gompertz, MMF (MORGAN et al., 1975), Chapman-Richards e Weibull; o modelo logístico foi escolhido e ajustado aos dados de cada estrato, utilizando-se o procedimento iterativo Levenberg-Marquardt, disponível no software SifProg 4.4 (2006). Um dos inconvenientes nesse tipo de modelagem é o fato de estimar, dentro de uma mesma idade, o mesmo volume, o que causa inconsistência para fins de regulação da produção. Isso implica muitas unidades de manejo com uma mesma expectativa de produção na idade de rotação regulatória e consequente limitação no modelo de regulação. Então, foi considerada a aplicação do coeficiente $\hat{V}_{i}=\hat{V}_{i} V_{u i} \hat{V}_{u i}^{-1}$ para fazer que a equação ajustada passasse pelos valores de volumes observados por ocasião do primeiro inventário.

A quarta alternativa (alternativa 4) foi a transformação de um modelo de predição em um modelo de projeção e posterior ajuste desse modelo por estrato de modelagem utilizando procedimentos de regressão não linear. $O$ modelo determinado foi o logístico, ficando definida a seguinte relação funcional:

$$
V_{2}=V_{1}+\hat{\beta_{0}}\left(1+\hat{\beta_{1}} e^{-\hat{\beta}_{2} I_{2}}\right)^{-1}-\hat{\beta_{0}}\left(1+\hat{\beta_{1}} e^{-\hat{\beta}_{2} I_{1}}\right)^{-1}+\varepsilon
$$

sendo as variáveis e parâmetros conforme já definidos. Esse sistema foi ajustado utilizando-se o procedimento Quasi-Newton disponível no software Statistica 7.0 (STATSOFT, 2007).

A quinta alternativa (alternativa 5) foi o ajuste do modelo sugerido em Campos e Leite (2006), da forma $L n V=\beta_{0}+\beta_{1} I^{-1} S^{-1}+\varepsilon$, sendo $I, S$ e $V$, conforme já definidos.

R. Árvore, Viçosa-MG, v.33, n.5, p.841-852, 2009 


\subsection{Modelagem volumétrica e análises de clones com menos de três medições}

Depois de definida a melhor alternativa para modelagem e prognose, foram analisadas as situações dos clones com menos de três medições, ou seja, foi estudado o problema da prognose para clones ou estratos com dados insuficientes para modelagem pelas alternativas 1 a 5 .

Para permitir comparações entre valores observados e estimados de volume, foram sorteados alguns clones entre os 37 e ora considerados como tendo uma medição, ora como tendo duas medições. O modelo logístico foi ajustado em cada um desses clones. Nos clones com apenas uma medição, foi utilizada a informação de volume do inventário e calculado o incremento médio anual (IMA) para a idade correspondente.

A partir das 37 equações de predição, foi possível estimar o volume e o IMA para a idade dos clones com uma medição (clones novos), ou seja, foram obtidas 37 estimativas de IMA dos clones comerciais, para a idade do clone novo.

O clone que apresentou a menor diferença de IMA quando comparado com o clone novo foi considerado o mais similar. Para fazer a prognose, foi usado o $M C P$ definido para o estrato do respectivo clone comercial. Nos clones que tinham duas medições, foi utilizada a informação da segunda medição de inventário.

\section{RESULTADOS E DISCUSSÃO}

\subsection{Estratificação para fins de modelagem do crescimento e da produção}

Com as estimativas dos parâmetros do modelo de Schumacher, $\operatorname{Ln} Y=\beta_{0}+\beta_{1} I^{-1}+\varepsilon$, obtidos para cada clone e para cada variável mensurada, efetuou-se a análise de agrupamento pelo método de Tocher, com base na distância euclidiana média, sendo definidos 15 estratos (Tabela 1). Nesse mesmo quadro constam as equações estimadas a partir da função logística, com $Y=H d$, visando estimar índices de local em cada estrato e considerando uma idadeíndice de 72 meses.

\subsection{Modelagem volumétrica e análises de clones com mais de três medições}

O ajuste do modelo de Clutter para ser usado na avaliação das alternativas 1 (Clutter sem aplicação de correção para área basal) e 2 (Clutter com a correção para área basal), é apresentado a seguir:

$$
\left\{\begin{array}{lr}
\operatorname{Ln}_{2}=\operatorname{Ln} B_{1}\left(\frac{I_{1}}{I_{2}}\right)+3,633418\left(1-\frac{I_{1}}{I_{2}}\right) & R^{2}=92,53 \% \\
\operatorname{Ln} V_{2}=1,815681-19,745510 \frac{1}{I_{2}}+0,017188 S+1,147794 \operatorname{Ln} B_{2}
\end{array}\right.
$$

Tabela 1 - Agrupamento dos 37 clones de eucalipto, considerando-se os oito parâmetros analisados e estimativas dos parâmetros do modelo

\begin{tabular}{|c|c|c|c|c|c|c|c|c|c|c|c|}
\hline Estrato & & \multicolumn{6}{|c|}{ Clones } & \multirow{2}{*}{$\frac{\hat{\beta}_{0}}{31,4223}$} & \multirow{2}{*}{$\frac{\hat{\beta}_{1}}{8,1877}$} & \multirow{2}{*}{$\frac{\hat{\beta}_{2}}{0,0930}$} & \multirow{2}{*}{$\frac{r_{\hat{y} y}}{0,9596}$} \\
\hline 1 & 36 & & & & & & & & & & \\
\hline 2 & 17 & & & & & & & 36,5225 & 3,5072 & 0,0310 & 0,9551 \\
\hline 3 & 13 & 21 & 22 & 25 & 28 & & & 35,3454 & 3,1442 & 0,0353 & 0,9505 \\
\hline 4 & 16 & 18 & & & & & & 36,6212 & 3,2395 & 0,0394 & 0,9397 \\
\hline 5 & 2 & 3 & 32 & & & & & 35,6624 & 3,4720 & 0,0510 & 0,9108 \\
\hline 6 & 30 & & & & & & & 34,5220 & 4,5660 & 0,0591 & 0,9029 \\
\hline 7 & 12 & & & & & & & 37,2981 & 2,7236 & 0,0352 & 0,9590 \\
\hline 8 & 14 & & & & & & & 31,6897 & 2,6274 & 0,0444 & 0,9174 \\
\hline 9 & 29 & & & & & & & 45,1014 & 4,9308 & 0,0374 & 0,9108 \\
\hline 10 & 4 & 5 & 6 & 31 & 33 & & & 33,5499 & 4,5936 & 0,0624 & 0,9287 \\
\hline 11 & 7 & 8 & 9 & 19 & 20 & 23 & 35 & 34,5472 & 3,5962 & 0,0517 & 0,9351 \\
\hline 12 & 11 & 15 & 24 & 26 & 27 & 37 & & 31,6813 & 4,0985 & 0,0618 & 0,9122 \\
\hline 13 & 10 & & & & & & & 34,8788 & 3,0021 & 0,0495 & 0,9279 \\
\hline 14 & 1 & & & & & & & 35,4865 & 2,8387 & 0,0435 & 0,9273 \\
\hline 15 & 34 & & & & & & & 33,6008 & 5,4129 & 0,0601 & 0,9659 \\
\hline
\end{tabular}

Table 1 - Grouping of the 37 eucalyptus clones, considering the 8 parameters analyzed and estimates of

R. Árvore, Viçosa-MG, v.33, n.5, p.841-852, 2009 
A variável $\left(1-\frac{I_{1}}{I_{2}}\right) S$ na expressão da área basal, foi retirada por apresentar valor não significativo estatisticamente. Resultados semelhantes foram encontrados por Dias et al. (2005), Valdez-Lazalde e Lynch (2000) e Bennet (1970).

Os ajustes dos modelos para avaliar as alternativas 3, 4 e 5 são apresentados na Tabela 2. A distribuição gráfica dos resíduos, $100(\hat{v}-v) v^{-1}$, calculados a partir das estimativas de volume de 2006, realizadas a partir da medição de 2005 (Figura 1), nas cinco alternativas, apresentou variações consideráveis, entre as alternativas nos respectivos estratos. O estrato 15 foi desconsiderado por não ter medição em 2006. A alternativa 4 foi a que apresentou a melhor distribuição dos resíduos, na maioria dos estratos. As alternativas 1 e 2 subestimaram o volume em todos os estratos, enquanto a alternativa 3 superestimou em locais com menor produtividade, ou idade inferior. Além disso, subestimou em locais de maior produtividade ou idades mais avançadas. A alternativa 5 apresentou a pior distribuição de resíduo.

As estimativas de volume obtidas a partir da medição de 2004 para 2006 apresentaram valores negativos para o estoque de volume total quando comparado com os valores observados. A alternativa 4 resultou em estimativas de estoque volumétrico mais próximo do valor observado do estoque total e, principalmente, nos estratos que possuem a maior área (estratos 3 a 5 e 10 a 13). Essas estimativas de volume também foram observadas nas projeções de 2005 para 2006. A estimativa do estoque total em 2006 , realizada a partir das medições das parcelas permanentes em 2005, apresentou exatidão de $-2,15 \%$, quando usada a alternativa quatro, o que é muito satisfatório em termos de manejo florestal. A alternativa quatro foi considerada a mais adequada para os dados analisados (Tabela 3 ).

É importante ressaltar que o processo de avaliação dos ajustes dos modelos deve ser contínuo, de forma a verificar discrepâncias significativas entre as estimativas desses modelos e a produção verdadeira (VANCLAY, 1994). Em condições de clima tropical, a atualização e consequente avaliação devem ser feitas, no caso de eucalipto, pelo menos a cada dois anos.

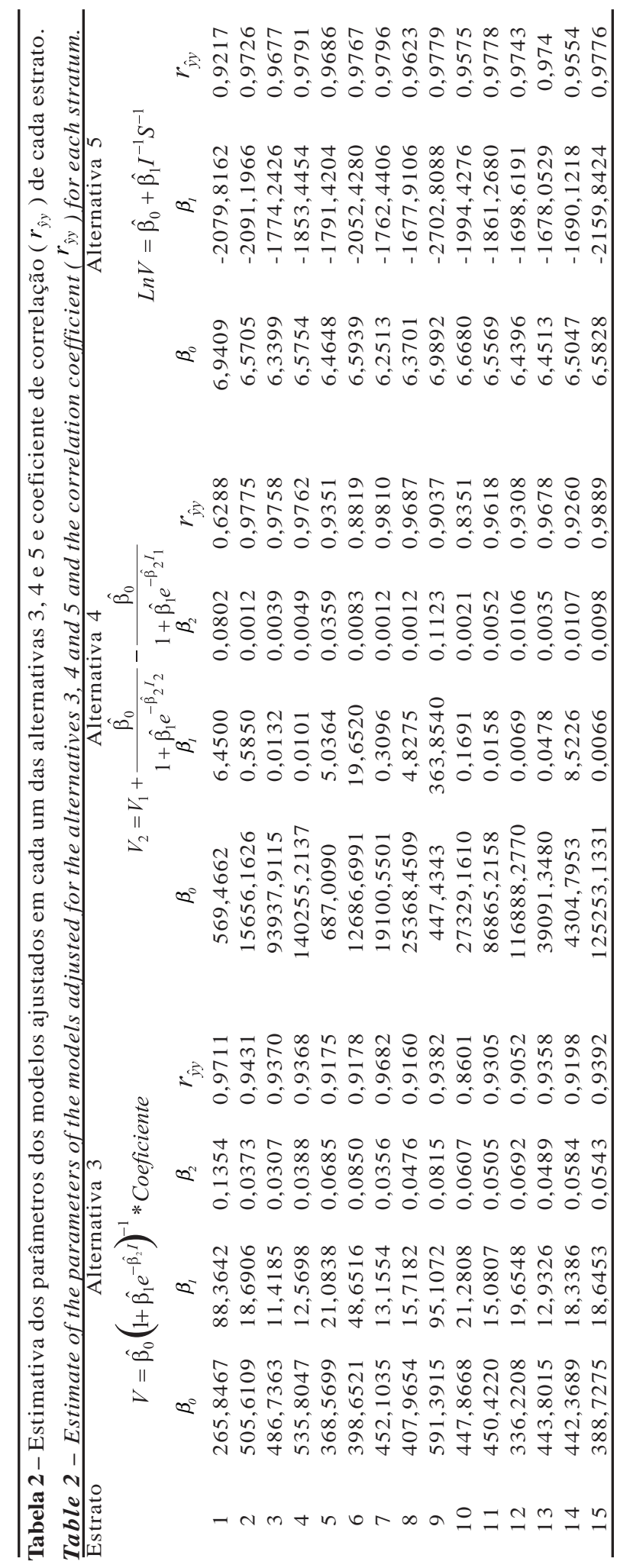

R. Árvore, Viçosa-MG, v.33, n.5, p.841-852, 2009 


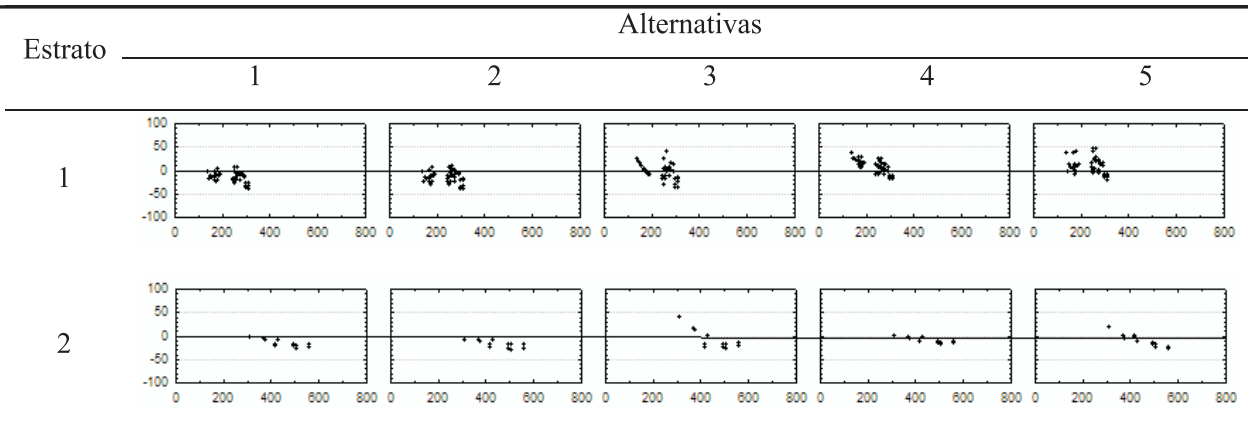

3

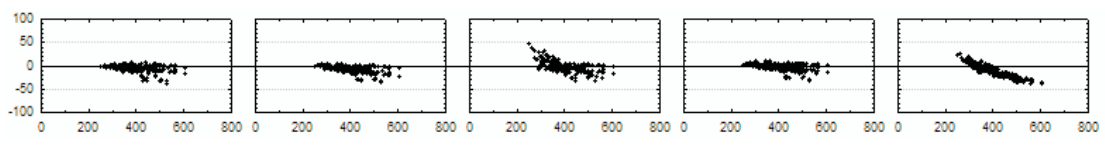

4

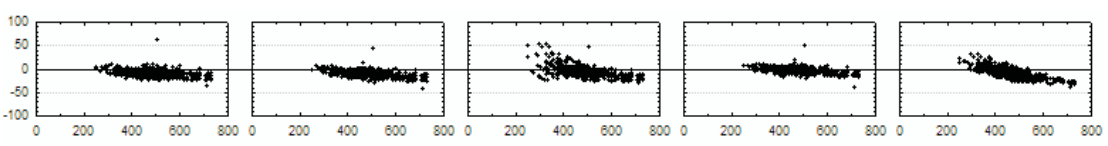

5

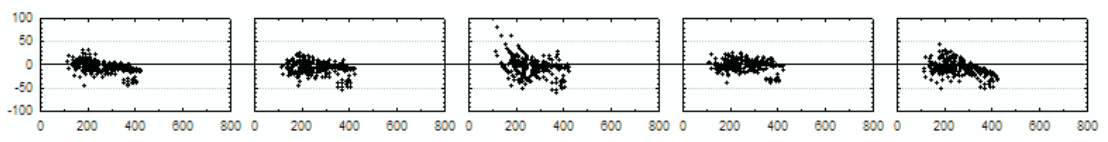

6
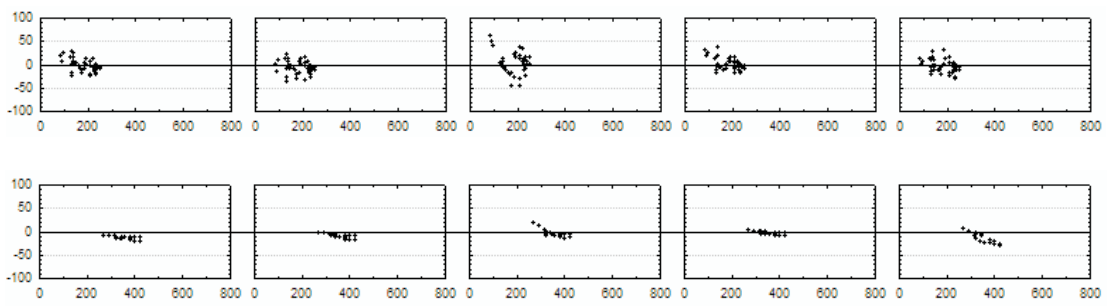

8

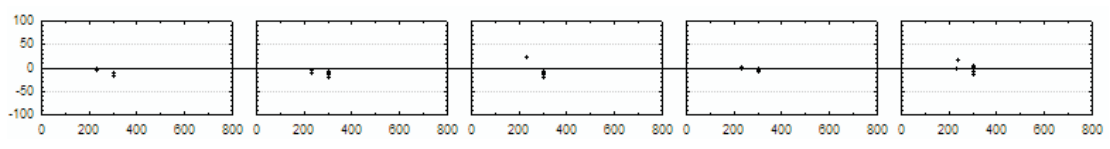

9

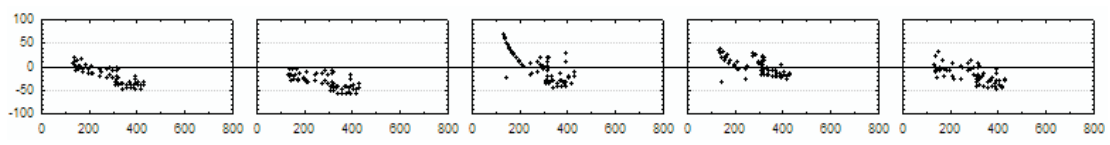

10

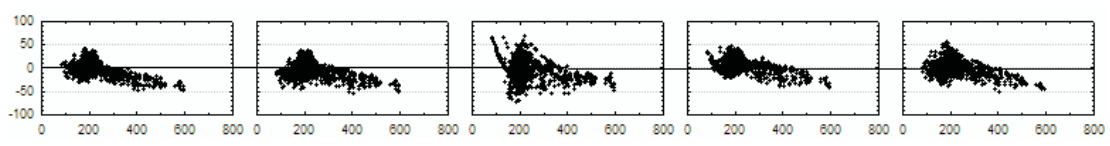

11

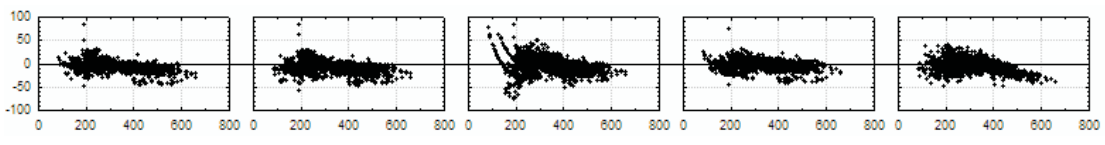

Figura 1 - Distribuição dos resíduos percentuais $\left(100(\hat{v}-v) v^{-1}\right)$ em cada estrato e em cada alternativa de avaliação. Figure 1 - Distribution of percentage residues $\left(100(\hat{v}-v) v^{-1}\right)$ for each stratum and each evaluation alternative.

R. Árvore, Viçosa-MG, v.33, n.5, p.841-852, 2009 
Tabela 3 -Estimativas do estoque de volume $\left(\mathrm{m}^{3}\right)$ do ano de 2006, obtidas a partir da medição das parcelas permanentes em 2004 e das medições de 2005 , área em ha e resíduo percentual $\left(100(\hat{v}-v) v^{-1}\right)$ por estrato e em cada alternativa de avaliação.

Table 3 - Estimates of volume stock $\left(\mathrm{m}^{3}\right)$ for 2006 obtained by measuring the permanent plots in 2004 and 2005 measurements, by area in ha and percentage residue $\left(100(\hat{v}-v) v^{-1}\right)$ per stratum and for each evaluation alternative.

\begin{tabular}{|c|c|c|c|c|c|c|c|c|c|c|c|c|}
\hline & & & & zadas a parti & nediç: & celas pe & nentes 1 & das em 2 & 004 & & & \\
\hline Estrato & Volume & Area (ha) & & Volume estima & em $2006\left(m^{3}\right)$ & Alternativa & & & Resíduc & (\%) por $\mathrm{Al}$ & rnativa & \\
\hline & $\begin{array}{c}\text { observado em } \\
2006\left(\mathrm{~m}^{3}\right)\end{array}$ & & 1 & 2 & 3 & 4 & 5 & 1 & 2 & 3 & 4 & 5 \\
\hline 1 & 4078,98 & 187,47 & 3340,25 & 3298,35 & 3747,62 & 4307,16 & 4423,18 & $-18,11$ & $-19,14$ & $-8,12$ & 5,59 & 8,44 \\
\hline 2 & 1847,50 & 104,12 & 1398,99 & 1347,68 & 1388,85 & 1576,59 & 1499,06 & $-24,28$ & $-27,05$ & $-24,83$ & $-14,66$ & $-18,86$ \\
\hline 3 & 34295,47 & 2093,78 & 30310,30 & 29434,66 & 30812,36 & 31794,07 & 28223,56 & $-11,62$ & $-14,17$ & $-10,16$ & $-7,29$ & $-17,70$ \\
\hline 4 & 110350,02 & 5943,61 & 94210,56 & 92904,77 & 99441,44 & 103906,89 & 93798,61 & $-14,63$ & $-15,81$ & $-9,89$ & $-5,84$ & $-15,00$ \\
\hline 5 & 26966,82 & 1734,48 & 23914,03 & 23608,50 & 23170,49 & 26090,87 & 23052,02 & $-11,32$ & $-12,45$ & $-14,08$ & $-3,25$ & $-14,52$ \\
\hline 6 & 2924,13 & 152,74 & 2763,95 & 2578,56 & 2721,02 & 2895,12 & 2677,25 & $-5,48$ & $-11,82$ & $-6,95$ & $-0,99$ & $-8,44$ \\
\hline 7 & 2188,62 & 34,83 & 1790,19 & 1858,01 & 1951,75 & 2047,22 & 1751,19 & $-18,20$ & $-15,11$ & $-10,82$ & $-6,46$ & $-19,99$ \\
\hline 8 & 576,62 & 34,48 & 490,24 & 473,19 & 476,32 & 545,10 & 531,45 & $-14,98$ & $-17,94$ & $-17,39$ & $-5,47$ & $-7,83$ \\
\hline 9 & 7010,88 & 521,71 & 4481,15 & 3768,68 & 5487,80 & 6760,31 & 4768,61 & $-36,08$ & $-46,25$ & $-21,72$ & $-3,57$ & $-31,98$ \\
\hline 10 & 78880,37 & 7595,55 & 68721,15 & 67247,33 & 60816,50 & 78413,22 & 72812,47 & $-12,88$ & $-14,75$ & $-22,90$ & $-0,59$ & $-7,69$ \\
\hline 11 & 259146,95 & 18853,99 & 225707,63 & 222850,10 & 223452,59 & 244519,71 & 226824,27 & $-12,90$ & $-14,01$ & $-13,77$ & $-5,64$ & $-12,47$ \\
\hline 12 & 90459,49 & 5964,50 & 77752,51 & 77118,75 & 69339,91 & 80833,62 & 78539,44 & $-14,05$ & $-14,75$ & $-23,35$ & $-10,64$ & $-13,18$ \\
\hline 13 & 35403,14 & 3350,12 & 30372,54 & 30730,61 & 30681,24 & 32960,80 & 31045,07 & $-14,21$ & $-13,20$ & $-13,34$ & $-6,90$ & $-12,31$ \\
\hline 14 & 3907,59 & 176,68 & 2944,46 & 2901,99 & 2977,47 & 3714,56 & 3172,43 & $-24,65$ & $-25,73$ & $-23,80$ & $-4,94$ & $-18,81$ \\
\hline Total & 658036,58 & 46748,06 & 568197,95 & 560121,20 & 556465,34 & 620365,25 & 573118,61 & $-13,65$ & $-14,88$ & $-15,44$ & $-5,72$ & $-12,90$ \\
\hline
\end{tabular}

\begin{tabular}{|c|c|c|c|c|c|c|c|c|c|c|c|c|}
\hline Estrato & $\begin{array}{c}\text { Volume } \\
\text { observado em } \\
2006\left(\mathrm{~m}^{3}\right)\end{array}$ & Area (ha) & \multicolumn{5}{|c|}{ Volume estimado em $2006\left(\mathrm{~m}^{3}\right)$ por Alternativa } & \multicolumn{5}{|c|}{ Resíduo (\%) por Alternativa } \\
\hline 1 & 4078,98 & 187,47 & 3391,52 & 3691,14 & 3743,45 & 3862,75 & 4334,11 & $-16,85$ & $-9,51$ & $-8,23$ & $-5,30$ & 6,25 \\
\hline 3 & 34295,47 & 2093,78 & 32663,45 & 32198,94 & 33000,76 & 33529,43 & 29059,92 & $-4,76$ & $-6,11$ & $-3,78$ & $-2,23$ & $-15,27$ \\
\hline 4 & 110350,02 & 5943,61 & 100918,70 & 100581,42 & 103890,86 & 106679,07 & 96070,70 & $-8,55$ & $-8,85$ & $-5,85$ & $-3,33$ & $-12,94$ \\
\hline 5 & 26966,82 & 1734,48 & 25451,27 & 25331,02 & 25760,56 & 26592,09 & 24966,22 & $-5,62$ & $-6,07$ & $-4,47$ & $-1,39$ & $-7,42$ \\
\hline 8 & 576,62 & 34,48 & 505,74 & 511,16 & 515,20 & 551,32 & 553,99 & $-12,29$ & $-11,35$ & $-10,65$ & $-4,39$ & $-3,92$ \\
\hline 9 & 7010,88 & 521,71 & 5091,64 & 4637,21 & 5803,40 & 6391,31 & 5713,51 & $-27,38$ & $-33,86$ & $-17,22$ & $-8,84$ & $-18,51$ \\
\hline 10 & 78880,37 & 7595,55 & 73509,13 & 74405,06 & 78797,31 & 79691,63 & 77825,90 & $-6,81$ & $-5,67$ & $-0,11$ & 1,03 & $-1,34$ \\
\hline 11 & 259146,95 & 18853,99 & 241708,55 & 243870,62 & 248151,12 & 255412,83 & 237609,74 & $-6,73$ & $-5,89$ & $-4,24$ & $-1,44$ & $-8,31$ \\
\hline 12 & 90459,49 & 5964,50 & 80897,19 & 85525,43 & 84364,54 & 86568,10 & 83006,97 & $-10,57$ & $-5,45$ & $-6,74$ & $-4,30$ & $-8,24$ \\
\hline 13 & 35403,14 & 3350,12 & 32170,91 & 33125,43 & 33704,39 & 34487,10 & 32096,47 & $-9,13$ & $-6,43$ & $-4,80$ & $-2,59$ & $-9,34$ \\
\hline 14 & 3907,59 & 176,68 & 2998,38 & 3057,95 & 3077,31 & 3528,59 & 3259,21 & $-23,27$ & $-21,74$ & $-21,25$ & $-9,70$ & $-16,59$ \\
\hline
\end{tabular}


Tabela 4-Incremento médio anual observado, estimativas de incremento médio anual e diferença percentual de duas alternativas de prognose, por idade, ano de medição e clone.

Table 4 - Annual mean increase observed, annual mean increase estimated, and percentage difference for two prognosis alternatives by age, year of measurement and clone.

\begin{tabular}{|c|c|c|c|c|c|c|c|c|c|c|c|}
\hline \multirow[t]{2}{*}{ Clone } & \multirow[t]{2}{*}{$\begin{array}{l}\text { Ano de } \\
\text { medição }\end{array}$} & \multirow[t]{2}{*}{$\begin{array}{l}\text { Idade } \\
\text { (meses) }\end{array}$} & \multirow[t]{2}{*}{$\begin{array}{l}I M A \\
\text { Obs }\end{array}$} & \multicolumn{4}{|c|}{$\begin{array}{l}\text { Alternativa } 4 \\
\frac{\hat{\beta_{0}}}{+\hat{\beta}_{1} e^{-\hat{\beta}_{2} I_{2}}}-\frac{\hat{\beta_{0}}}{1+\hat{\beta}_{1} e^{-\hat{\beta}}}\end{array}$} & \multicolumn{4}{|c|}{$\begin{array}{c}\text { Alternativa } 1 \\
\text { Modelo de Clutter sem correção }\end{array}$} \\
\hline & & & & $\begin{array}{l}M A \text { est a } \\
\text { partir de } 24\end{array}$ & $\begin{array}{c}\text { Diferença } \\
(\%)\end{array}$ & $\begin{array}{c}I M A \text { est a } \\
\text { partir de } 36\end{array}$ & $\begin{array}{c}\text { Diferença } \\
(\%)\end{array}$ & $\begin{array}{l}I M A \text { est a } \\
\text { partir de }\end{array}$ & $\begin{array}{c}\text { Diferença } \\
(\%)\end{array}$ & $\begin{array}{l}I M A \text { est } \\
\text { a partir }\end{array}$ & $\begin{array}{c}\text { Diferença } \\
(\%)\end{array}$ \\
\hline \multirow[t]{5}{*}{1} & 2002 & 24 & 41,4 & - & - & - & - & - & - & - & - \\
\hline & 2003 & 36 & 51,2 & 55,0 & 7,5 & - & - & 56,0 & 9,4 & - & - \\
\hline & 2004 & 48 & 58,0 & 61,4 & 5,8 & 55,2 & $-4,9$ & 59,5 & 2,5 & 57,3 & $-1,3$ \\
\hline & 2005 & 60 & 61,2 & 65,0 & 6,2 & 56,7 & $-7,3$ & 58,7 & $-4,1$ & 58,1 & $-5,1$ \\
\hline & 2006 & 72 & 80,4 & 67,1 & $-16,6$ & 57,1 & $-29,0$ & 56,3 & $-30,0$ & 56,3 & $-30,1$ \\
\hline \multirow[t]{5}{*}{2} & 2002 & 24 & 34,9 & - & - & - & - & - & - & - & - \\
\hline & 2003 & 36 & 44,4 & 46,7 & 5,3 & - & - & 50,8 & 14,4 & - & - \\
\hline & 2004 & 48 & 49,4 & 51,4 & 4,1 & 51,0 & 3,3 & 55,5 & 12,3 & 50,4 & 2,2 \\
\hline & 2005 & 60 & 54,0 & 52,6 & $-2,5$ & 53,4 & $-1,1$ & 55,6 & 3,1 & 53,3 & $-1,3$ \\
\hline & 2006 & 72 & 53,3 & 52,4 & $-1,7$ & 54,3 & 1,8 & 54,0 & 1,4 & 52,4 & $-1,7$ \\
\hline \multirow[t]{5}{*}{3} & 2002 & 24 & 34,2 & - & - & - & - & - & - & - & - \\
\hline & 2003 & 36 & 47,5 & 44,8 & $-5,7$ & - & - & 50,2 & 5,7 & - & - \\
\hline & 2004 & 48 & 52,8 & 51,6 & $-2,2$ & 52,6 & $-0,3$ & 54,9 & 4,0 & 52,0 & $-1,4$ \\
\hline & 2005 & 60 & 57,5 & 57,0 & $-0,8$ & 56,5 & $-1,8$ & 55,1 & $-4,0$ & 55,5 & $-3,5$ \\
\hline & 2006 & 72 & 56,8 & 61,6 & 8,5 & 57,7 & 1,5 & 53,4 & $-6,0$ & 54,1 & $-4,7$ \\
\hline \multirow[t]{5}{*}{4} & 2002 & 24 & 31,6 & - & - & - & - & - & - & - & - \\
\hline & 2003 & 36 & 39,9 & 39,1 & $-2,2$ & - & - & 47,8 & 19,6 & - & - \\
\hline & 2004 & 48 & 49,2 & 42,9 & $-12,8$ & 50,3 & 2,3 & 53,3 & 8,3 & 46,8 & $-4,9$ \\
\hline & 2005 & 60 & 55,4 & 45,3 & $-18,2$ & 52,8 & $-4,6$ & 54,0 & $-2,4$ & 49,0 & $-11,5$ \\
\hline & 2006 & 72 & 53,2 & 46,9 & $-11,7$ & 53,9 & 1,4 & 52,9 & $-0,6$ & 48,6 & $-8,5$ \\
\hline \multirow[t]{5}{*}{6} & 2002 & 24 & 35,9 & - & - & - & - & - & - & - & - \\
\hline & 2003 & 36 & 50,0 & 48,7 & $-2,6$ & - & - & 52,9 & 5,8 & - & - \\
\hline & 2004 & 48 & 55,9 & 52,9 & $-5,4$ & 52,5 & $-6,1$ & 57,7 & 3,2 & 54,1 & $-3,1$ \\
\hline & 2005 & 60 & 59,9 & 53,8 & $-10,2$ & 54,6 & $-8,9$ & 57,8 & $-3,6$ & 57,0 & $-4,8$ \\
\hline & 2006 & 72 & 80,7 & 53,3 & $-34,0$ & 55,3 & $-31,5$ & 55,9 & $-30,8$ & 55,5 & $-31,3$ \\
\hline \multirow[t]{5}{*}{7} & 2002 & 24 & 37,7 & - & - & - & - & - & - & - & - \\
\hline & 2003 & 36 & 50,4 & 49,9 & $-1,0$ & - & - & 52,7 & 4,7 & - & - \\
\hline & 2004 & 48 & 52,3 & 53,8 & 2,9 & 53,8 & 2,9 & 57,1 & 9,3 & 55,2 & 5,6 \\
\hline & 2005 & 60 & 60,5 & 54,5 & $-9,9$ & 54,5 & $-9,9$ & 57,1 & $-5,7$ & 57,9 & $-4,3$ \\
\hline & 2006 & 72 & 60,8 & 54,0 & $-11,3$ & 54,0 & $-11,3$ & 55,3 & $-9,0$ & 56,5 & $-7,1$ \\
\hline \multirow[t]{5}{*}{8} & 2002 & 24 & 29,4 & - & - & - & - & - & - & - & - \\
\hline & 2003 & 36 & 40,0 & 37,1 & $-7,1$ & - & - & 46,2 & 15,6 & - & - \\
\hline & 2004 & 48 & 48,8 & 40,9 & $-16,0$ & 49,6 & 1,8 & 51,8 & 6,2 & 47,1 & $-3,5$ \\
\hline & 2005 & 60 & 53,0 & 43,2 & $-18,6$ & 51,2 & $-3,4$ & 52,7 & $-0,6$ & 49,9 & $-5,8$ \\
\hline & 2006 & 72 & 72,8 & 44,5 & $-38,9$ & 51,2 & $-29,7$ & 51,7 & $-29,0$ & 49,6 & $-31,8$ \\
\hline \multirow[t]{5}{*}{10} & 2002 & 24 & 36,2 & - & - & - & - & - & - & - & - \\
\hline & 2003 & 36 & 43,4 & 48,9 & 12,6 & - & - & 46,6 & 9,6 & - & - \\
\hline & 2004 & 48 & 46,1 & 53,0 & 15,0 & 53,6 & 16,2 & 51,8 & 13,9 & 45,8 & 0,8 \\
\hline & 2005 & 60 & 52,8 & 53,9 & 2,2 & 57,2 & 8,4 & 52,5 & 0,7 & 48,8 & $-6,4$ \\
\hline & 2006 & 72 & 54,0 & 53,5 & $-1,0$ & 58,3 & 8,0 & 51,4 & $-3,3$ & 48,6 & $-8,6$ \\
\hline \multirow[t]{4}{*}{11} & 2002 & 24 & 40,1 & - & - & - & - & - & - & - & - \\
\hline & 2003 & 36 & 56,1 & 49,6 & $-11,6$ & - & - & 55,6 & $-0,9$ & - & - \\
\hline & 2004 & 48 & 60,4 & 55,5 & $-8,1$ & 60,8 & 0,6 & 59,5 & $-1,5$ & 58,0 & $-4,0$ \\
\hline & 2005 & 60 & 62,0 & 58,8 & $-5,1$ & 64,5 & 4,1 & 59,0 & $-4,7$ & 60,1 & $-2,9$ \\
\hline
\end{tabular}

R. Árvore, Viçosa-MG, v.33, n.5, p.841-852, 2009 
Tabela 4 - Cont.

\begin{tabular}{|c|c|c|c|c|c|c|c|c|c|c|c|}
\hline & 2006 & 72 & 61,6 & $59, \overline{7}$ & $-3, \overline{1}$ & 66,6 & $\overline{8,1}$ & 56,8 & $-7,7$ & 58,0 & $-5,8$ \\
\hline \multirow[t]{5}{*}{16} & 2002 & 24 & 32,9 & - & - & - & - & - & - & - & - \\
\hline & 2003 & 36 & 45,2 & 43,9 & $-2,9$ & - & - & 48,1 & 6,4 & - & - \\
\hline & 2004 & 48 & 61,9 & 50,9 & $-17,8$ & 51,9 & $-16,1$ & 53,3 & $-13,9$ & 53,4 & $-13,8$ \\
\hline & 2005 & 60 & 64,0 & 56,5 & $-11,8$ & 55,9 & $-12,7$ & 53,9 & $-15,8$ & 55,1 & $-13,9$ \\
\hline & 2006 & 72 & 69,9 & 61,0 & $-12,7$ & 57,3 & $-18,1$ & 52,6 & $-24,8$ & 54,1 & $-22,6$ \\
\hline \multirow[t]{5}{*}{19} & 2002 & 24 & 37,6 & - & - & - & - & - & - & - & - \\
\hline & 2003 & 36 & 44,0 & 52,5 & 19,3 & - & - & 53,7 & 22,0 & - & - \\
\hline & 2004 & 48 & 56,3 & 59,6 & 5,8 & 53,3 & $-5,3$ & 58,1 & 3,2 & 56,2 & $-0,2$ \\
\hline & 2005 & 60 & 63,8 & 63,5 & $-0,4$ & 58,4 & $-8,5$ & 58,0 & $-9,1$ & 58,9 & $-7,7$ \\
\hline & 2006 & 72 & 59,7 & 65,9 & 10,4 & 62,9 & 5,4 & 55,8 & $-6,4$ & 56,9 & $-4,6$ \\
\hline \multirow[t]{5}{*}{20} & 2002 & 24 & 31,4 & - & - & - & - & - & - & - & - \\
\hline & 2003 & 36 & 41,6 & 45,7 & 9,8 & - & - & 48,5 & 16,7 & - & - \\
\hline & 2004 & 48 & 47,6 & 50,6 & 6,2 & 51,2 & 7,5 & 53,3 & 11,9 & 50,1 & 5,1 \\
\hline & 2005 & 60 & 51,0 & 52,0 & 1,9 & 55,3 & 8,3 & 53,6 & 5,1 & 52,0 & 1,8 \\
\hline & 2006 & 72 & 48,4 & 51,8 & 7,1 & 56,8 & 17,2 & 52,2 & 7,9 & 51,2 & 5,7 \\
\hline
\end{tabular}

\subsection{Modelagem volumétrica e análises de clones com menos de três medições}

Para fazer as comparações das estimativas de volume dos clones com uma e duas medições, foram selecionadas as unidades de prognose que tinham 24 meses em 2002, ou seja, tinham a primeira medição nesse ano 2002, e foram medidas em todos os anos até 2006, totalizando cinco medições. Com isso foram selecionados 12 clones (Tabela 4). Esses clones tinham mais de duas medições, mas, para servir de base comparativa, o procedimento foi realizado com a própria base de dados que tinha 37 clones comerciais. Para isso, esses 12 clones foram considerados ora como tendo medição, ora como tendo duas medições.

Com os coeficientes da equação logística ajustado para cada clone, foi estimado o volume e calculado o incremento médio anual (IMA) para a idade de 24 meses (primeira medição) e para 36 meses (segunda medição) para os 37 clones. Depois disso, foi determinado qual clone apresentava a menor diferença de IMA e, portanto, definido em qual estrato o novo clone faria parte. Esse procedimento foi realizado em 12 clones determinados.

No caso de uma só medição e para melhor entendimento, tome-se como exemplo o clone 1 . Inicialmente, o clone 1 foi eliminado da base dos clones comerciais e considerado apenas como uma medição. O IMA do clone 1 foi de $41,4 \mathrm{~m}^{3} \cdot \mathrm{ha}^{-1}$.ano ${ }^{-1}$ aos 24 meses (Tabela 4); com a determinação do clone comercial com o IMA mais próximo deste valor, foi verificado a qual estrato de modelagem esse clone pertencia e usado o modelo desse estrato para fazer a prognose do clone 1 , para idades variando de 36 a 72 meses.
No caso do clone com duas medições, foi usada a segunda medição para comparação. No mesmo clone 1 , o IMA aos 36 meses é de 51,2 $\mathrm{m}^{3}$.ha $\mathrm{h}^{-1}$.ano ${ }^{-1}$, seguindo-se o mesmo procedimento para o clone com uma medição, só que agora foi comparada com as estimativas de IMA aos 36 meses dos outros 36 clones comerciais.

Para fazer a prognose dos casos de clones com uma ou duas medições, foi considerada a alternativa 4. O objetivo foi verificar, no caso de clones com menos de três medições, se é melhor um modelo mais simples (com menos variáveis) por estrato ou um modelo mais complexo (com mais variáveis) ajustado com todos os dados. Não foi possível determinar qual a melhor alternativa, já que em alguns clones a melhor resposta foi dada pelo modelo mais simples (alternativa 4), enquanto em outros o melhor foi a estimativa usando o modelo de Clutter, ajustado com todos os dados (Tabela 4). Em alguns casos, a melhor alternativa mudou ao considerar uma ou duas medições. Portanto, no caso de clones com menos de três medições a escolha de uma alternativa deve ser feita pelo modelador e pelo usuário, e as expectativas do usuário não devem ser desconsideradas.

Em clones que não possuem nenhuma medição, a solução pode ser o uso de uma curva de produção média gerada com dados de determinada região ou estrato. Nesse caso, a experiência do usuário é fundamental. Essa recomendação é semelhante àquela proposta por Campos e Leite (2006).

A modelagem do crescimento e da produção é um processo dinâmico e sempre que necessário deve ser atualizada, pelo menos a cada dois anos. Os bancos

R. Árvore, Viçosa-MG, v.33, n.5, p.841-852, 2009 
de dados de parcelas permanentes devem ser atualizados e consistidos para ajustes de modelos de crescimento e produção e para a classificação da capacidade produtiva. A amostragem com a finalidade de avaliar a capacidade produtiva e a modelagem do crescimento e da produção deve ser representativa. Sempre que possível, é melhor utilizar um número menor de parcelas, porém representando toda a área em termos de classe de produtividade, do que um número muito grande de parcelas, porém sem representatividade de condições extremas e intermediárias. Em banco de dados de inventário florestal contínuo (IFC), isso não ocorre. Muitas vezes, as condições extremas não são representadas de modo eficiente e sempre haverá excesso de informações na condição média. É preciso ressaltar que a modelagem não visa representar uma condição média como o IFC. Isso resulta na necessidade de amostragem seletiva, no banco de dados de inventário, para fins de modelagem. Além disso, em alguns casos é necessário lançar parcelas específicas para captar variações nas condições extremas.

Procedimentos utilizados atualmente no Brasil para classificação da capacidade produtiva e modelagem precisam ser revistos, já que grande parte desses estudos não tem considerado a dinâmica dos plantios clonais. Alguns desses procedimentos muitas vezes foram desenvolvidos no Brasil e até em outros países, para plantios de sementes, ou para outras condições de ambiente.

O modelo de Clutter (1963), um dos mais difundidos no Brasil (CAMPOS e LEITE, 2006), foi ajustado originalmente com dados de Pinus taeda, nos EstadosO modelo de Clutter (1963), um dos mais difundidos no Brasil (CAMPOS e LEITE, 2006), foi ajustado originalmente com dados de Pinus taeda, nos Estados Unidos. No Brasil, esse modelo tem sido usado na forma definida pelo autor, para uma condição específica. O próprio autor chamava a atenção que o sistema definido naquela ocasião não seria aplicado a todas as espécies e nem a todos os tipos de manejo. O modelo original era composto da seguinte relação funcional para estimar o volume:

$L n V=\beta_{0}+\beta_{1} S+\beta_{2}(\operatorname{Ln} B)+\beta_{3} \frac{1}{\mathrm{I}}+\beta_{4} S(\operatorname{Ln} B)+\beta_{5} S \frac{1}{\mathrm{I}}+\beta_{6} \frac{1}{\mathrm{I}}(\operatorname{Ln} B)+\varepsilon$

R. Árvore, Viçosa-MG, v.33, n.5, p.841-852, 2009
Com base na significância dos parâmetros, em dados de P. taeda o modelo foi reduzido ao sistema representado pelas equações 1 e 2 do item 2.3 .

As expressões 1 e 2 sempre foram utilizadas no Brasil e, na maioria dos casos, têm resultado em estimativas precisas e livres de viés. Entretanto, principalmente em povoamentos de clones de eucalipto, é necessário avaliar a contribuição estatística de cada uma das variáveis exógenas do sistema.

Além da modelagem, é necessária atenção especial ao realizar a prognose em povoamentos clonais. Um modelo pode estar muito bem ajustado e validado, porém, se seu uso não for correto, pode resultar em sérios equívocos nos modelos de regulação florestal.

Com o avanço dos recursos computacionais, a realização da prognose tornou-se tarefa menos trabalhosa, ficando a maior parte do trabalho na escolha dos procedimentos mais adequados para dada espécie, região, regime de corte etc., já que a simulação utilizando esses procedimentos é rápida. Apesar disso, a prognose ainda continua sendo grande desafio para o setor florestal.

A modelagem deve ser um processo interativo entre modelador e usuário. Para as empresas não é interessante que o usuário seja o modelador, pela possibilidade de adição de tendências nas estimativas; no entanto o modelador também não deveria assumir o papel de usuário, já que na maioria das vezes ele não tem conhecimento específico sobre a área de estudo e, até mesmo, sobre as expectativas de produtividade.

\section{CONCLUSÕES}

O procedimento proposto neste estudo para modelagem do crescimento e produção de clones com mais de três medições é eficiente.

Na região de estudo e no período contemplado, a alternativa 4 , determinada pelo uso do modelo

$$
V_{2}=V_{1}+\hat{\beta_{0}}\left(1+\hat{\beta_{1}} e^{-\hat{\beta}_{2} I_{2}}\right)^{-1}-\hat{\beta_{0}}\left(1+\hat{\beta_{1}} e^{-\hat{\beta}_{2} I_{1}}\right)^{-1}
$$

por estrato, foi mais eficiente para estimar o estoque volumétrico total.

A estimativa de crescimento e produção de clones que possuem menos de três medições pode ser realizada através de modelos específicos por estrato ou de um 
modelo geral, porém essa decisão deve ser avaliada melhor pelo modelador e pelo usuário.

Procedimentos de modelagem de plantios de clones de eucalipto devem ser revistos continuamente.

\section{REFERÊNCIAS}

AVERY, T. E.; BURKHART, H. E. Forest measurements. 4.ed. New York: McGraw-Hill Book, 1994. 408p.

BENNET, F. A. Variable-density yield tables for managed stands of natural slash pine. USDA, Forestry Service, 1970. 7p. (Research note - SE-141)

CAMPOS, J. C. C.; CAMPOS, A. L. A. S.; LEITE, H. G. Decisão silvicultural empregando um sistema de predição do crescimento e da produção.

Revista Árvore, v.12, n.2, p.100-110, 1988.

CAMPOS, J. C. C. et al. Aplicação de um modelo compatível de crescimento e produção de densidade variável em plantações de Eucalyptus grandis. Revista Árvore, v.10, n.2, p.121-134, 1986.

CAMPOS, J. C. C.; LEITE, H. G. Mensuração florestal: perguntas e respostas. 2.ed. Viçosa, MG: Universidade Federal de Viçosa, 2006. 470p.

CLUTTER, J. L. Compatible growth and yield for loblolly pine. Forest Science, v.9, n.3, p.354371, 1963.

ClUtTER, J. L. et al. Timber management: a quantitative approach. New York: John Willey \& Sons, 1983. 333p.

CURTIS, R. O.; MARSHALL, D. D. Permanente-plot procedures for silvicultural and yield research. Washington: United States Department of Agriculture, Forest Service, Pacific Northwest Research Station., 2005. 86p.

DIAS, A. N. et al. Emprego de um modelo de crescimento e produção em povoamentos desbastados de eucalipto. Revista Árvore, v.29, n.5, p.731-739, 2005.
DRAPER, N. R.; SMITH, H. Applied regression analysis. 3.ed. New York: John Willey \& Sons, 1998. 706p.

GARCIA, S. L. R.; LEITE, H. G. Curso de estatística florestal. Viçosa, MG: Faculdade de Viçosa, 2006. 401p. (Não Publicado).

GUIMARÃES, D. P. Desenvolvimento de um modelo de distribuição diamétrica de passo invariante para prognose e projeção da estrutura de povoamentos de eucalipto. 1994. 160f. Tese (Doutorado em Ciência Florestal) - Universidade Federal de Viçosa, Viçosa-MG, 1994.

LEITE, H. G. Ajuste de um modelo de estimação de frequência e produção por classe de diâmetro, para povoamentos de Eucalyptus saligna Smith.1990. 78f. Dissertação (Mestrado em Ciência Florestal) - Universidade Federal de Viçosa, Viçosa, MG, 1990.

MORGAN, P. H.; MERCER, L. P.; FLODIN, N. W. General model for nutrition responses of higher organisms. The Proceedings of the National Academy of Sciences of the United States of America, v.72, n.11, p.4327-4331, 1975.

NOGUEIRA, G. S. et al. Modelo de distribuição diamétrica para povoamentos de Eucalyptus sp. submetidos a desbaste. Revista Árvore, v.29, n.4, p.579-589, 2005.

PECE DE RIOS, M. G. V. Um modelo de crescimento e produção aplicado a plantações de Eucalyptus pellita. 1993. 75 f. Dissertação (Mestrado em Ciência Florestal) Universidade Federal de Viçosa, Viçosa, MG, 1993.

ROSAS, M. P. Alternativas de determinação da idade técnica de corte de Eucalyptus urophylla. 1994. $70 f$. Dissertação (Mestrado em Ciência Florestal) Universidade Federal de Viçosa, Viçosa, MG, 1994.

SCHUMACHER, F. X. A new growth curve and its application to timber studies. Journal of Forestry, v.37, NUMERO, p.819-820, 1939.

R. Árvore, Viçosa-MG, v.33, n.5, p.841-852, 2009 
SIFPROG. Sistema para elaboração de estudos de prognose. Versão 4.4. 2006.

<www.treesoftware.com.br>

TREVIZOL JÚNiOR, T. L. Análise de um modelo compatível de crescimento e produção em plantações de Eucalyptus grandis (W.Hill ex-Maiden). 1985. 74f.

Dissertação (Mestrado em Ciência Florestal) Universidade Federal de Viçosa, Viçosa, MG, 1985.
STATSOFT, INC. Statistica (data analysis software system), version 7. 2007. <www.statsoft.com>

VALDEZ-LAZALDE, J. R.; LYNCH, T. B.

Merchantable and total volume equations for thinned stands of Patula Pine in Puebla, México. Agrociência, v.34. n.6, p.747-758, 2000.

VANCLAY, J.K. Modelling forest growth and yield. Wallingford, UK, CAB International, 1994. $312 \mathrm{p}$ 